\title{
Clinicopathological Features of Gastroenteropancreatic Neuroendocrine Neoplasms
}

\author{
Abdusselam Sekerci ${ }^{1}$, Haci Mehmet Turk², Tarik Demir ${ }^{2}$, Mesut Seker ${ }^{2}$, Adem Akcakaya ${ }^{3}$ and Dilek Sema Arici ${ }^{4}$ \\ ${ }^{1}$ Department of Internal Medicine, Faculty of Medicine, Bezmialem Vakif University, Turkey \\ ${ }^{2}$ Department of Medical Oncology, Faculty of Medicine, Bezmialem Vakif University, Turkey \\ ${ }^{3}$ Department of General Surgery, Faculty of Medicine, Bezmialem Vakif University, Turkey \\ ${ }^{4}$ Department of Pathology, Faculty of Medicine, Bezmialem Vakif University, Turkey
}

\begin{abstract}
Objective: To describe the clinical, pathological and survival characteristics of Turkish patients with gastroenteropancreatic neuroendocrine neoplasms (GEP-NENs).

Study Design: Descriptive study.

Place and Duration of Study: Bezmialem Vakif University School of Medicine Hospital from April 2012 to September 2018.

Methodology: Sixty-one patients with GEP-NENs were analysed in retrospect for the clinical, pathological and survival characteristics. The Kaplan-Meier method was used for survival analysis and the Log-rank test was performed to analyse the comparisons between the groups.

Results: Forty-five of the 61 GEP-NENs patients (73.8\%) were neuroendocrine tumor (NET) and $26.2 \%$ of patients were neuroendocrine carcinoma (NEC). The mean age of patients was $55.5 \pm 11.3$ years. The most frequent localisation of tumors was stomach (34.4\%), and the most common symptom was abdominal pain (27.9\%). The rate of distant metastases was $31.1 \%$ at diagnosis and $63.9 \%$ of the patients were operated. The median follow-up period was 27 months. The rate of three-years overall survival (OS) was $88.5 \%$ and the five-years OS rate was $86.9 \%$. The variables that can significantly influence the OS rate were high grade (grade 3; $p=0.005)$, Ki-67 proliferation index (Ki-67 $>20 \% ; p=0.002)$ and distant metastases $(p=0.018)$.

Conclusion: GEP-NENs may develop anywhere in the digestive tract. Approximately one-third of the patients may be metastatic due to delay in diagnosis.
\end{abstract}

Key Words: Gastroenteropancreatic neuroendocrine tumor, Neuroendocrine cancers, Neuroendocrine neoplasms.

How to cite this article: Sekerci A, Turk HM, Demir T, Seker M, Akcakaya A, Arici DS. Clinicopathological Features of Gastroenteropancreatic Neuroendocrine Neoplasms. J Coll Physicians Surg Pak 2020; 30(08):863-867.

\section{INTRODUCTION}

Neuroendocrine neoplasms (NENs) are uncommon tumors and have heterogeneous clinical behaviour. NENs may occur in different sites of the body. GEP-NENs, originating from the gastrointestinal tract and pancreas, consist of more than half of NENs. ${ }^{1,2}$ The incidence is about $3.65-4.7$ per 100000 . The incidence of NENs has been increasing over time, this rising may be due to increased diagnosis on radiographic imaging and common use of endoscopy. ${ }^{3}$ Neuroendocrine cells are specialised cells capable of producing, storing and secreting peptide and biogenic amines.

Correspondence to: Abdusselam Sekerci, Department of Internal Medicine, Faculty of Medicine, Bezmialem Vakif University, Turkey

E-mail:dr.asekerci@gmail.com

Received: January 24, 2020; Revised: May 17, 2020;

Accepted: June 13, 2020

DOI: https://doi.org/10.29271/jcpsp.2020.08.863
If gastroenteropancreatic neuroendocrine tumors (GEP-NETs) cause neuroendocrine syndromes due to overexpression of a peptide-structured hormone, they are called as functional GEPNET. If they don't cause a clinical picture, they are called as non-functional GEP-NET. ${ }^{4,5}$ Especially in non-functional cases, diagnosis can last up to 5 years. For this reason, $60 \%$ of patients are inoperable when admitted to the hospital. ${ }^{6}$

The proliferative activity of tumorcells provides important prognostic information in NENs. To this end, grading is performed with the cell proliferation rate. Ki-67 cell proliferation index and mitotic activity indicates the rate of proliferation. GEP-NENs were categorised as well differentiated neuroendocrine tumors (G1 and G2) and poorly differentiated neuroendocrine carcinomas (G3) in 2010 World Health Organization (WHO) classification. ${ }^{7.8}$

The aim of the present study was to describe the clinicopathologic and survival characteristics of patients with GEP-NEN.

\section{METHODOLOGY}

This retrospective descriptive study was approved by the Clinical Research Ethics Committee of Bezmialem Vakif University 
(approval number:14/281, dated 16.07.2019). The hospital records of GEP-NENs patients were reviewed retrospectively. Clinical, pathological and survival features of patients admitted to Bezmialem Vakif University School of Medicine Hospital, from April 2012 to September 2018 were analysed. Collected information included clinical characteristics (age, sex, symptoms, and location of tumors), pathological properties (grade, size, metastases, histopathology of tumor), the treatments and follow-up process. GEP-NENs are pathologically diagnosed by the morphological and immunohistochemical features.

The pathologic diagnosis of GEP-NENs established in accordance with the WHO 2010 classification. Grading was depended on tumor morphology and proliferative activity. According to the Ki-67 index; Ki-67 index $\leq 2 \%$, 3-20\% and $>20 \%$ were grouped as G1, G2 and G3, respectively. Similarly, according to mitotic rates; tumors with mitotic rates $<2,2-20$ and $>20$ in 10 high-power fields were grouped as $\mathrm{G} 1, \mathrm{G} 2$, and $\mathrm{G} 3$, respectively. When the grading of the Ki-67 index differed from that of the mitotic rate, categorised by high value. GEP-NENs were classified as well differentiated neuroendocrine tumor (NET) (G1 and G2) or poorly differentiated neuroendocrine carcinoma (NEC) (G3) according to WHO 2010 classification.

Various imaging methods were used to diagnosis and staging of GEP-NENs, such as computed tomography (CT), magnetic resonance imaging (MRI), endoscopy, endoscopic ultrasound (EUS) and positron emission tomography (PET-CT, using with ${ }^{18} \mathrm{~F}$ FDG $/{ }^{68} \mathrm{Ga}$ Dotatate). Tomography is a frequently used imaging modality. ${ }^{18} \mathrm{~F}$-FDG PET/CT varies handy for staging especially in poorly differentiated NENs. Whereas ${ }^{68} \mathrm{Ga}$ Dotatate PET/CT was performed on well and intermediate differentiated NENs. MRI is used in diagnosis of pancreas and liver mass.

SPSS 20.0 for Windows (IBM Corporation. Armonk, NY, USA) was used for statistical analyses. Quantitative data were expressed as the amount or range. Qualitative data were expressed in a name, symbol or number code. OS was defined as the interval between diagnosis and death. İdentification of independent risk factors for tumor metastasis was performed by Multivariate Cox regression analysis. Kaplan-Meier method was used to draw survival plots, and Log-rank test was applied to compute differences between subgroups. P-values less than 0.05 were assumed as statistically significant.

\section{RESULTS}

The clinical and pathological features of the patients are shown in Tables I and II. Among 61 patients, 25 (41\%) were men and 36 $(59 \%)$ were women; the ratio of female to male was 1.44 . Age ranged from 28 to 77 years and the mean age was $55.5( \pm 11.3)$ years. The most frequent tumor localisation was the stomach (34.4\%), followed by the pancreas $(21.3 \%)$, unknown primary origin $(11.5 \%)$, duodenum $(8.2 \%)$, colon $(4.9 \%)$, rectum $(4.9 \%)$, appendix (4.9\%) and other sites including periampullary, jejunum/ileum and cholecyst. The vast majority of GEP-NENs $(96.7 \%)$ was non-functional; the other two patients $(3.3 \%)$ presented symptoms of carcinoid syndrome such as flushing, tachycardia, and hypertension. The $37.7 \%$ of the tumors were grade $1,36.1 \%$ grade 2 and $26.2 \%$ grade 3 . Atimmunohistochem- ical staining, the positive rates of synaptophysin, chromogranin, and CD56 were $77 \%, 80.3 \%$, and $24.6 \%$, respectively.

Table I. Clinical features of the patients.

\begin{tabular}{|l|l|c|}
\hline \multicolumn{2}{|l|}{ Parameter } & Nean \pm S.D \\
\hline Age & Female & $55.5 \pm 11.3$ \\
\hline \multirow{2}{*}{ Sex } & Male & $36(59)$ \\
& Abdominal pain & $17(41)$ \\
\hline & Nausea & $7(27.9)$ \\
Symptoms & Weight loss & $4(6.6)$ \\
& Vomiting & $2(3.3)$ \\
& Hypertension & $2(3.3)$ \\
& Flushing-Tachycardia & $2(3.3)$ \\
\hline \multirow{2}{*}{ Functional status } & Non-functional & $59(96.7)$ \\
& Functional & $2(3.3)$ \\
\hline \multirow{2}{*}{ Distant metastasis } & & $23(37.7)$ \\
\hline \multirow{3}{*}{ Metastatic site } & & $17(27.9$ \\
& Liver & $3(4.9)$ \\
& Lung & $7(11.5)$ \\
& Lymph node & $3(4.9)$ \\
\hline
\end{tabular}

Tablell. Pathological features of the patients.

\begin{tabular}{|l|l|c|}
\hline \multicolumn{2}{|l|}{ Parameter } & N (\%) \\
\hline \multirow{4}{*}{ Tumor localisation } & Stomach & $21(34.4)$ \\
& Pancreas & $13(21.3)$ \\
& Unknown primary origin & $7(11.5)$ \\
& Duodenum & $5(8.2)$ \\
& Colon & $3(4.9)$ \\
& Rectum & $3(4.9)$ \\
& Appendix & $3(4.9)$ \\
& Periampullary & $2(3.3)$ \\
& Jejunum-ileum & $2(3.3)$ \\
& Cholecyst & $2(3.3)$ \\
\hline \multirow{2}{*}{ Grade } & G1 & $23(37.7)$ \\
& G2 & $22(36.1)$ \\
\hline \multirow{2}{*}{ Immunohistochemistry } & G3 & $16(26.2)$ \\
(positivity rates) & Synaptophysin & $47(77)$ \\
& Chromogranin & $49(80.3)$ \\
& CD 56 & $15(24.6)$ \\
\hline
\end{tabular}

The most common initial complaint was abdominal pain (27.9\%), followed by nausea (11.5\%), weight loss (6.6\%), abdominal distention (3.3\%) and other symptoms. Three patients (4.9\%) were diagnosed during a routine physical examination. Distant metastases were found in $31.1 \%$ (19/61) of patients at initial diagnosis, but the number increased to $37.7 \%(23 / 61)$ during the follow-up. Although the most common metastasis occurs in the liver, there were also patients with lymph node, lung and bone metastases. Three tumors located in the pancreas were found by EUS-guided fine-needle biopsy, while the tumors initially detected in the liver.

Thirty-nine patients (63.9\%) were operated to curative intent. Sixteen patients received chemotherapy and the mostly used chemotherapeutic agents were cisplatin-etoposide. Six patients treated with octreotide and four patients received lanreotide as somatostatin analogues. Hepatic arterial chemoembolization and radiofrequency ablation were used in two of the patients. One patient underwent liver transplantation and one patient received palliative radiotherapy (RT) to theliver. 


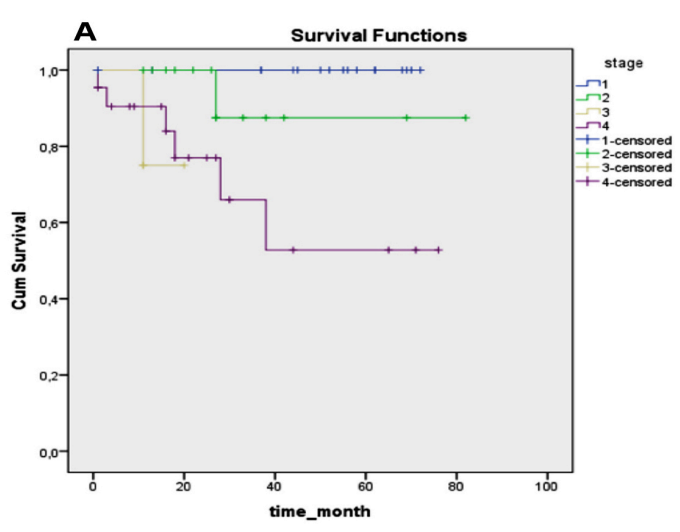

Sixty-one patients were clinically followed for periods of 2-78 months. The median survival time could not be reached because of the short follow-up time and small number of deaths in patients with well-differentiated GEP-NET. Three-years OS rate was $88.5 \%$, and 5 years OS rate was $86.9 \%$. The factors that could significantly influence the $O S$ high grade (grade $3 ; p=$ 0.005 , Figure $1 \mathrm{~A})$, distant metastasis ( $p=0.018$, Figure $1 \mathrm{~B})$, stage $(p=0.001$, Figure $1 \mathrm{C}$ ) and Ki-67 proliferation index (Ki-67 $>20 \% ; p=0.002$, Figure 1D). No statistically significantrelationship was found between age $(p=0.199)$, gender $(p=0.271)$, tumor diameter $(p=0.089)$ and OS.

\section{DISCUSSION}
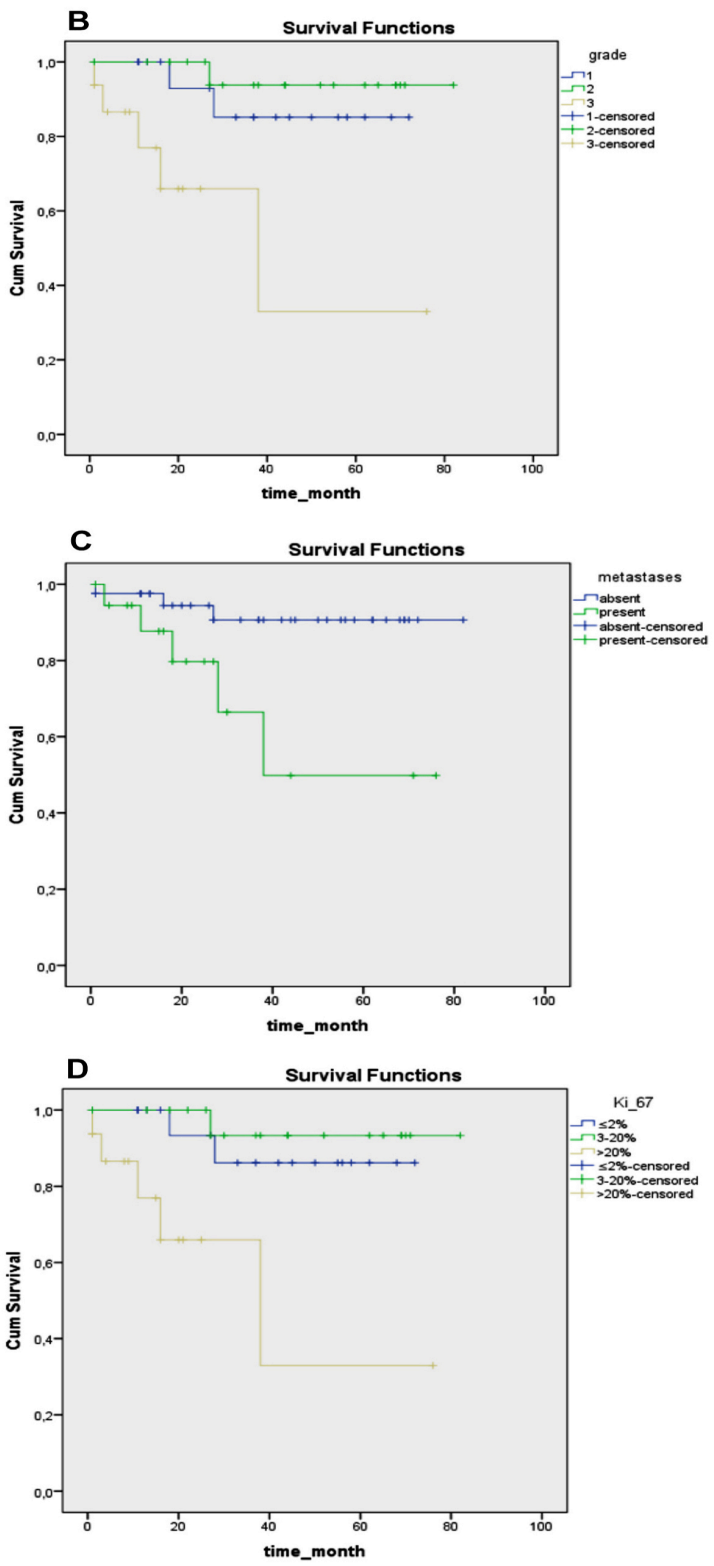

Figure 1. Overall survival curves stratified by stage (A), grade (B), metastases (C), and Ki67 (D).

NENs tend to occur mostly in the gastrointestinal tract but they may appear anywhere in the body. ${ }^{9}$ Overall, in terms of prognostic factors, non-distant metastases, lower tumor grade and Ki-67 proliferation were associated with better survival. The present retrospective study aim to assess the clinical, survival and pathological characteristics of GEP-NENs patients in the study centre.

It was found that the most frequent localisation was the stomach, followed by the pancreas, and duodenum. In a Chinese study, the most common site was stomach, too. Whereas, analysis from the Surveillance, Epidemiology, and End of Results (SEER) database $(n=29664)$ detected that the rectum was the highest frequent location of GEP-NENs. ${ }^{10}$ The different distribution of GEP-NENs in some geographical regions may be due to different reasons. The higher incidence in the rectum may be due to the use of screening colonoscopy. Sometimes, small gastric NENs encountered during endoscopy may not be considered malignant. In our study, only a few of cases were in the colon. The rate of distant metastases was $37.7 \%$. This rate was higher than the United States and was close to rates in Spain. ${ }^{11,12}$

GEP-NENs are divided into two groups according to whether they are symptomatic. Functional tumors have symptomatic and associated with overproduction of peptides and hormones. Our study showed that GEP-NENs were mostly non-functional tumors. Diagnosis in non-functional tumors that occult is usually later.

Even in metastatic patients, the primary treatment of the GEP-NENs is surgical treatment to palliation and increase survival. If possible, removal of both primary focus and metastasis is essential. ${ }^{13}$ Ablative treatments, such as radiofrequency ablation (RFA) or transarterial chemoembolisation, can be used to treat liver metastasis. There are no randomised trials to investigate the superiority of one ablative therapy over another. ${ }^{14}$ On the other hand, poorly differentiated advanced stage GEP-NENs are treated with chemotherapy. The most frequently used chemotherapy in the treatment of GEP-NENs is cisplatin and etoposide combination like small cell lung cancer treatment. ${ }^{15}$ The treatment recommendations for metastatic low-grade GEP-NETs as chemotherapy include streptozocin based regimens (streptozocin+fluorouracil/doxorubicin) or temozolo- 
mide with or without capecitabine. ${ }^{16-18}$ Somatostatin analogues are especially useful in improving hormone-related symptoms. Somatostatin analogues, such as lanreotide and octreotide, significantly prolonged progression-free survival (PFS) in patients with well differentiated metastatic GEP-NETs. ${ }^{19,20}$ Also, ${ }^{177}$ Lu-Dotatate for midgut neuroendocrine tumors (NETTER-1) has been resulted in markedly longer progression-free survival than high-dose octreotide LAR in patients who had progressive disease on somatostatinanalogue. ${ }^{21}$

In this study, 39 patients had surgery, including radical and palliative surgical treatment. RFA was applied to the liver of two patients. We used cisplatin/carboplatin- etoposide in the first line treatment for 10 neuroendocrine cancer patients, similar to small cell lung cancer treatment. Three patients with metastatic pancreas NET (PNET) used temozolomide (40 $\mathrm{mg} /$ day). Four patients used capecitabine $\left(1650 \mathrm{mg} / \mathrm{m}^{2} /\right.$ day $)$. Ten patients underwent somatostatin analogues. After the NETTER-1 study published, three well-differentiated metastatic midgutpatients weregiven ${ }^{177}$ Lutecium treatmentafterprogression with somatostatin analogues. All patients completed the four cycles of ${ }^{177}$ Lutecium. Although one patient had progression at tenth months, two patients were continued as progression-free for 12 and 15 months. Two patients developed grade 1 hematological toxicity during ${ }^{177}$ Lutecium treatment.

A pancreatic NEC patient, who had exhausted all treatment options, had right upper quadrant pain due to diffuse liver metastasis. Despite all palliative interventions, the patient's pain did not decrease. Although not in routine practice, we gave palliative RT to the liver of the patient and there was a significant decrease in the patient's symptoms. No liver toxicity related to RT was observed.

Liver transplantation can be used to treat patients with metastatic NETs in selected cases. Liver transplantation is a choice of treatment in liver metastatic PNETs, especially in symptomatic patients with unresectable metastasis. ${ }^{22}$ The results of retrospective European Liver Transplant Registry study of 213 cases undergoing liver transplantation for NETs between 1982 and 2009 were reported in 2013 by Le Traut et al. This study found that the overall survival reaches $80 \%$ if the patient selection is done with strict criteria. ${ }^{23}$ In this study, liver transplantation was performed for one patient after everolimus treatment. Here, patient with grade 2 PNET metastatic to the liver at presentation were treated with a defined strategy. After a short term everolimus therapy, the resection to pancreatic primary and liver transplantation were successfully performed in September 2015. The patient had a living donor-liver transplantation from his wife, and resection of the pancreatic primary at the same surgical session was conducted. The patient was still alive without progression and had been in a good health for 27 months. Everolimus efficacy data comes from the phase 3 randomised Radiant-3 trial, which everolimus demonstrated a significant prolonged PFS compared to best supportive care. ${ }^{24}$

This study has some limitations. First, the follow-up period of this study was not enough to reach the median survival time.
This may be due to short follow-up time and to the fact of slow growth rate of the NENs. Second, the number of patients was low for a comprehensive evaluation.

\section{CONCLUSION}

GEP-NENs may develop anywhere in the gastrointestinal tract. Most of the patients have non-functional tumors; and the fact that approximately one-third of the patients have metastatic may be due to delay in diagnosis. These results about GEP-NETs will increase the knowledge of physicians and result in earlier diagnosis of the disease.

\section{ETHICALAPPROVAL:}

The Clinical Research Ethics Committee of Bezmialem Vakif University approved the study. We obtained the ethical approvals prior to initiation of the research work.

\section{PATIENTS' CONSENT:}

This study is retrospective and covers the years 2012-2018. It is often difficult to reach patients of previous 7-8 years because some of these patients do nothave contact information, some of them have been expired. For these reasons, informed consent could not be obtained from patients.

\section{CONFLICT OF INTEREST:}

The authors declare that there is no conflict of interest.

\section{AUTHORS' CONTRIBUTION:}

AS: Conception, design, supervision, resources, materials, data collection and processing, analysis and interpretation, literature search, writing manuscript, critical review

HMT: Conception, design, supervision, resources, materials, data collection and processing, literature search, writing manuscript, critical review

TD: Design, supervision, resources, materials, data collection and processing, literature search, writing manuscript, critical review

MS, AA, DSA: Resources, materials, data collection and processing.

\section{REFERENCES}

1. Díez M, Teulé A, Salazar R. Gastroenteropancreatic neuroendocrine tumors: Diagnosis and treatment. Ann Gastroenterol 2013; 26(1):29-36.

2. Karpathakis A, Caplin M, Thirlwell C. Hitting the target: Where do molecularly targeted therapies fit in the treatment scheduling of neuroendocrine tumours? Endocr Relat Cancer 2012; 19(3):R73-92.

3. Hallet J, Law CH, Cukier M, Saskin R, Liu N, Singh S. Exploring the rising incidence of neuroendocrine tumors: $A$ population-based analysis of epidemiology, metastatic presentation, and outcomes. Cancer 2015; 121(4):589-97.

4. Robert TJ. Endocrine tumors of the gastrointestinal tract and pancreas. Harrison's Principles of Internal Medicine. 18th edition ed2013 p.3056-72.

5. Kaltsas GA, Besser GM, Grossman AB. The diagnosis and medical management of advanced neuroendocrine tumors. Endocr Rev 2004; 25(3):458-511. 
6. Oberg K. Neuroendocrine tumors of the digestive tract: Impact of new classifications and new agents on therapeutic approaches. Curr Opin Oncol 2012; 24(4): 433-40.

7. Bosman FT. WHO classification of tumours of the digestive system. 4th ed. Lyon: International Agency for Research on Cancer; 2010.

8. Falconi M, Bettini R, Scarpa A, Capelli P, Pederzoli P. Surgical strategy in the treatment of gastrointestinal neuroendocrine tumours. Ann Oncol 2001; 12 (Suppl 2): S101-3.

9. Modlin IM, Oberg K, Chung DC, Jensen RT, de Herder WW, Thakker RV, et al. Gastroenteropancreatic neuroendocrine tumours. Lancet Oncol 2008; 9(1):61-72.

10. Lawrence B, Gustafsson BI, Chan A, Svejda B, Kidd M, Modlin IM. The epidemiology of gastroenteropancreatic neuroendocrine tumors. Endocrinol Metab Clin North Am 2011; 40(1):1-18, vii.

11. Metz DC, Jensen RT. Gastrointestinal neuroendocrine tumors: Pancreatic endocrine tumors. Gastroenterology 2008; 135(5):1469-92.

12. Garcia Carbonero R, Capdevila J, Crespo Herrero G, Diaz Perez JA, Martinez Del Prado MP, et al. Incidence patterns of care and prognostic factors for outcome of gastroenteropancreatic neuroendocrine tumors (GEP-NETs): Results from the National Cancer Registry of Spain (RGETNE). Ann Oncol 2010; 21(9):1794-803.

13. Garcia-Carbonero R, Salazar R, Sevilla I, Isla D. SEOM clinical guidelines for the diagnosis and treatment of gastroenteropancreatic neuroendocrine tumours (GEP NETS). Clin Transl Oncol 2011; 13(8):545-51.

14. Rindi G, Wiedenmann B. Neuroendocrine neoplasms of the gut and pancreas: New insights. Nature Reviews Endocrinology 2012; 8(1):54-64.

15. Yamaguchi T, Machida N, Morizane C, Kasuga A, Takahashi $\mathrm{H}$, Sudo $\mathrm{K}$, et al. Multicenter retrospective analysis of systemic chemotherapy for advanced neuroendocrine carcinoma of the digestive system. Cancer Sci 2014; 105(9):1176-81.
16. Moertel CG, Lefkopoulo M, Lipsitz S, Hahn RG, Klaassen D. Streptozocin-doxorubicin, streptozocin-fluorouracil or chlorozotocin in the treatment of advanced islet-cell carcinoma. N Engl J Med 1992; 326(8):519-23.

17. Dilz LM, Denecke T, Steffen IG, Prasad V, Von weikersthal LF, Pape UF, et al. Streptozocin/5-fluorouracil chemotherapy is associated with durable response in patients with advanced pancreatic neuroendocrine tumours. Eur J Cancer 2015; 51(10):1253-62.

18. Strosberg JR, Fine RL, Choi J, Nasir A, Coppola D, Chen DT, et al. First-line chemotherapy with capecitabine and temozolomide in patients with metastatic pancreatic endocrine carcinomas. Cancer 2011; 117(2):268-75.

19. Rinke A, Muller $\mathrm{HH}$, Schade-Brittinger $\mathrm{C}$, Klose KJ, Barth $\mathrm{P}$, Wied $M$, et al. Placebo-controlled, double-blind, prospective, randomised study on the effect of octreotide LAR in the control of tumor growth in patients with metastatic neuroendocrine midgut tumors: A report from the PROMID Study Group. J Clin Oncol 2009; 27(28): 4656-63.

20. Caplin ME, Pavel M, Cwikla JB, Phan AT, Raderer M, Sedlackova $E$, et al. Lanreotide in metastatic enteropancreatic neuroendocrine tumors. N Engl J Med 2014; 371(3):224-33.

21. Strosberg J, El-Haddad G, Wolin E, Hendifar A, Yao J, Chasen $B$, et al. Phase 3 Trial of (177)Lu-Dotatate for Midgut Neuroendocrine Tumors. N Engl J Med 2017; 376(2): 125-35.

22. Lehnert T. Liver transplantation for metastatic neuroendocrine carcinoma: An analysis of 103 patients. Transplantation 1998; 66(10):1307-12.

23. Le Treut YP, Gregoire E, Klempnauer J, Belghiti J, Jouve E, Lerut J, et al. Liver transplantation for neuroendocrine tumors in Europe-results and trends in patient selection: A 213-case European liver transplant registry study. Ann Surg 2013; 257(5):807-15.

24. Yao JC, Shah MH, Ito T, Bohas CL, Wolin EM, Van Cutsem E, et al. Everolimus for advanced pancreatic neuroendocrine tumors. N Engl J Med 2011; 364(6):514-23. 